DOI: 10.3901/JME.2015.03.129

\title{
复小波共振解调频带优化方法和新指标*
}

\author{
张 龙 熊国良 黄文艺 \\ (华东交通大学机电工程学院 南昌 330013)
}

\begin{abstract}
摘要: 滚动轴承发生局部故障时, 其振动信号中将出现周期性发生的脉冲响应。共振解调被广泛用于从振动信号中提取此类 冲击响应特征, 但其首要问题是确定带通滤波器的中心频率和带宽。现有的频带优化方法存在计算量大且优化指标只考虑信 号中冲击成分的强弱、而未考虑脉冲发生的周期性特征等缺点。针对不足, 确定了以中心频率为主带宽为辅的频带参数优化 方法, 并提出了一个既能反映信号中冲击成分的存在及强弱又能突出冲击发生的周期性特点的频带优化新指标一一包络谱峰 值因子, 该因子定义为包络谱最大值与有效值之比。将此方法和新指标应用于复平移 Morlet 小波共振解调的频带优化, 通 过轴承疲劳试验数据分析及工程实际应用并与峭度、平滑度指标和山农摘进行比较, 验证了该方法和指标在最优频带确定中 的有效性和优越性。
\end{abstract}

关键词: 共振解调; 小波滤波; Morlet 小波; 频带优化

中图分类号: TN132

\section{New Procedure and Index for the Parameter Optimization of Complex Wavelet Based Resonance Demodulation}

\author{
ZHANG Long XIONG Guoliang HUANG Wenyi \\ (School of Mechatronics Engineering, East China Jiaotong University, Nanchang 330013)
}

\begin{abstract}
Local defects in bearings give rise to periodic impulses in vibrations. Resonance demodulation is widely adopted to extract such impulse train from vibrations. In order for the resonance demodulation to be used to good effect, various optimization procedure and index have been introduced to optimize the frequency band of resonance demodulation. Unfortunately, most of the optimization procedures available are computationally intensive, whilst the indices only depict the existence and level of an impact without considering its periodical occurrence. Targeted at such deficiencies, a procedure which searches for optimal center frequency with a high resolution while bandwidth with a low resolution is presented so as to alleviate computation burden. Moreover, a new index termed crest factor of envelope spectrum is formulated as the ratio of the maximum value to the root mean square of envelope spectrum, taking into account both the level and periodicity of impulses. The proposed procedure and index is employed to optimize the parameters of complex shifted Morlet wavelet for bearing defect diagnosis, and results on bearing run-to-failure experiment and filed data are in favorable of them in comparison with kurtosis, smoothness index and Shannon entropy.
\end{abstract}

Key words: resonance demodulation; wavelet filter; Morlet wavelet; frequency band optimization

\section{0 前言}

滚动轴承是旋转机械中使用最广泛也是最易 发生故障的零部件之一，有效的滚动轴承状态监测 与故障诊断方法一直是人们研究的目标。当轴承元 件表面出现点蚀、剥落、裂纹或压痕等局部损伤类

* 国家自然科学基金(51205130，51265010)、江西省教育厅(GJJ12318)和 江西省自然科学基金(20132BAB216029)资助项目。20140322 收到初 稿, 20141015 收到修改稿
故障时, 在轴承运行过程中损伤点会撞击与之接触 的其他元件表面而产生冲击力，该冲击力作用时间 短、频带宽, 会激励起轴承、轴承座、传感器等相 关结构的共振, 由于阻尼作用这个共振通常会在下 一次冲击力到来之前衰减掉, 从而在传感器测得的 振动信号中形成一个由许多脉冲响应(阻尼衰减振 动)构成的脉冲链 ${ }^{[1-2]}$ 。利用共振解调(或称为包络解 调、高频共振)从复杂的振动信号中提取脉冲响应 特征是广为采用的旋转机械循环冲击类故障检测 
方法。

鉴于小波变换优良的局部时频特性和滤波特 性, 连续小波变换(Continuous wavelet transform, $\mathrm{CWT})^{[3-5]}$ 和离散小波变换(Discrete wavelet transfrom, DWT $)^{[6-8]}$ 均被用作共振解调的滤波器。但是 DWT 对频域进行二进划分, 不够灵活和精细, 难于保证 振动信号中的共振区域刚好落在其中一个频段, 所 以效果也就不能得到保证。CWT 虽然是一种冗余的 信号表示方法, 但其连续变化的尺度参数却恰恰给 小波滤波器的设计带来了灵活性, 因此 CWT 在共 振解调中得到了广泛的应用 ${ }^{[9]}$ 。CWT 中用到的基函 数如 Morlet 小波具有与轴承局部故障引起的冲击衰 减类似的波形, 因而能够较好地提取淹没在噪声中 的冲击类故障特征 ${ }^{[10-12]}$ 。从频域看, Morlet 小波的 频谱具有窗口形式, 所以 CWT 的实质是利用一系 列性质相同但中心频率和带宽不同的滤波器(由母 小波通过平移和拉伸获得)对被分析信号进行滤波。 就共振解调而言, 其通常只需 CWT 在某一个中心 频率和带宽上的滤波结果, 而不需要所有尺度, 因 此如何确定小波共振解调的中心频率和带宽参数关 乎特征提取效果的好坏。许多学者在这方面做出了 有益的工作, 如 LIN 等 ${ }^{[9]}$ 将控制小波函数中心频率 和带宽的两个参数的取值范围分别离散为 40 和 30 个点, 然后计算总共 $30 \times 40=1200$ 种不同参数组合 所得滤波信号的峭度并选择峭度值最大的组合作为 最优小波参数。BOZCHALOOI 等 ${ }^{[13]}$ 针对滚动轴承 故障诊断提出了一个称为平滑度指标的新优化指 标, 并以步长 $50 \mathrm{~Hz}$ 在 [200 Hz, $6000 \mathrm{~Hz}$ ] 范围内 搜索小波函数最优中心频率, 以 0.01 的步长在 $[0.01$, 1]范围内搜索最优带宽参数, 因此总共有 $117 \times 100=$ 11700 种参数组合。可见同时进行两个参数的优化 将导致搜索空间和计算量过大, 不适合仪器实现和 现场使用。为此, $\mathrm{HE}$ 等 ${ }^{[14]}$ 以滤波后信号峭度最大 为目标函数, 利用启发式算法进行最优参数搜索, 大大提高了搜索效率。然而, 所采用的微分进化算 法本身存在参数人为选择的主观性, 也增加了整个 算法的复杂度。

目前带通滤波器参数的确定存在两个主要问 题: (1) 同时优化两个参数导致搜索空间过大, 效率 降低; (2) 所采用的优化指标如峭度、平滑度和山 农熵都只能度量信号的脉冲性(稀疏性)而未考虑 轴承局部故障引起的冲击响应周期性发生的特点, 这是故障冲击区别于外界干扰引入的偶然性冲击 的关键。为此, 本文分析了中心频率和带宽参数对 滤波效果的影响, 以决定主要优化对象、减小搜索 空间; 并提出了一个能够同时考虑冲击信号强度和
周期性强弱的频带优化新指标。最后利用轴承疲劳 试验数据和工程实际案例对所提方法和指标进行 了验证。

\section{1 复平移小波滤波}

\section{1 连续小波变换}

母函数 $\Psi(t)$ 经平移和尺度伸缩得到小波函数系 $\left\{\Psi_{\tau, a}(t)\right\}$, 其形式为

$$
\Psi_{\tau, a}(t)=\frac{1}{\sqrt{a}} \Psi\left(\frac{t-\tau}{a}\right)
$$

式中， $\tau$ 为时移； $a$ 为尺度因子 $(a>0) ; 1 / \sqrt{a}$ 用于实 现小波能量的归一化。定义一个能量有限信号 $x(t)$ 的小波变换为

$$
\begin{array}{r}
W T(a, \tau)=\left\langle x(\tau), \Psi_{\tau, a}^{*}(t)\right\rangle= \\
\frac{1}{\sqrt{a}} \int_{-\infty}^{\infty} x(t) \Psi^{*}\left(\frac{t-\tau}{a}\right) \mathrm{d} t
\end{array}
$$

式中, $\Psi^{*}{ }_{\tau, a}(t)$ 是 $\Psi_{\tau, a}(t)$ 的共轭。

\section{2 复平移 Morlet 小波滤波解调}

小波基函数的选择取决于被分析信号性质和 应用目的。在已有的小波函数中，Morlet 小波具有 指数衰减型震荡形式, 与轴承故障产生的冲击振动 响应成分十分相似, 因此 Morlet 小波在滚动轴承共 振解调技术方面得到了广泛研究 ${ }^{[3-5,9,14]}$ 。Morlet 复 小波定义为高斯函数与复指数函数的乘积 ${ }^{[14]}$

$$
\Psi(t)=\frac{\sigma}{\sqrt{\pi}} \exp \left(-\sigma^{2} t^{2}\right) \exp \left(\mathrm{i} 2 \pi f_{0} t\right)
$$

式中, 频率 $f_{0}$ 决定小波震荡快慢, 参数 $\sigma$ 决定小波 衰减速度。Morlet 小波的频域表示如下

$$
\Psi(f)=\exp \left[-\frac{\pi^{2}}{\sigma^{2}}\left(f-f_{0}\right)^{2}\right]
$$

式中, $\Psi(f)$ 是实函数, 有 $\Psi(f)=\Psi^{*}(f)$ 。在频域 中, $\sigma$ 控制小波窗口宽度, $f_{0}$ 控制窗口中心位置。

Morlet 小波频域为高斯窗口形式, 定义其半功 率带宽为幅值等于 $\sqrt{2} / 2$ 倍最大幅值所对应的上下 截止频率之间的宽度。式(4)所对应的小波的半功滤 率带宽 $\beta$ 为

$$
\beta=\frac{\sqrt{2 \ln 2}}{\pi} \sigma
$$

从而可知 Morlet 小波滤波器等效于一个通带为 $\left[f_{0}-\beta / 2, f_{0}+\beta / 2\right]$ 的带通滤波器。用带宽参数 $\beta$ 代替式 (4)中的 $\sigma$, Morlet 小波的频域表达式可以写为 


$$
\Psi(f)=\exp \left[-\frac{2 \ln 2}{\beta^{2}}\left(f-f_{0}\right)^{2}\right]
$$

通过选择不同带宽 $\beta$ 和对中心频率 $f_{0}$ 进行平移 可得到不同的滤波器, 同时因为 Morlet 小波的时域 函数为复函数, 所以这种小波滤波被称为复平移小 波滤波 ${ }^{[5,14-15]}$ 。以 $f_{0}=0.25 \mathrm{~Hz}, \beta=0.03 \mathrm{~Hz}$ 为例, Morlet 小波时域波形实部和频域波形分别如图 $1 \mathrm{a}$ 和图 $1 \mathrm{~b}$ 所示, 并给出了半功率带宽所对应的上下截止频率, 可以看出 Morlet 小波频谱具有窗口形式。

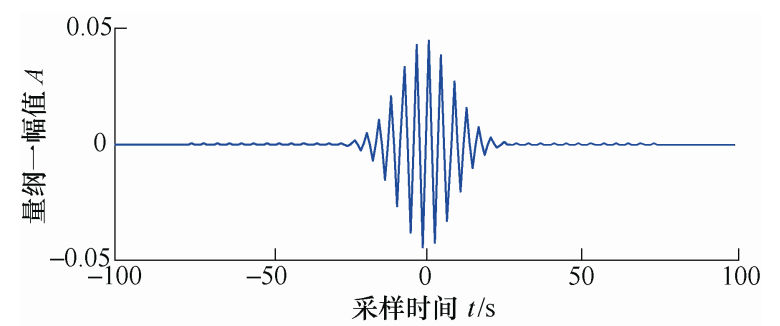

(a) 小波函数实部时域波形

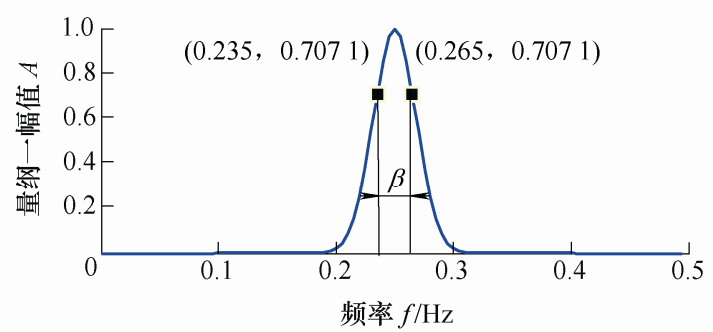

(b) 小波函数频谱

图 1 Morlet 小波时域和频域波形

由式(2)可知, 连续小波变换本质上是求不同尺 度的小波与被分析信号之间的卷积。根据时域卷积 定理, 小波滤波过程可以采用频域相乘的方式进行

$$
W T\left(f_{0}, \beta\right)=\mathrm{F}^{-1}\left[x(f) \Psi^{*}(f)\right]
$$

式中, $\mathrm{F}^{-1}$ 表示傅里叶反变换。根据 Hilbert 变换的 性质, 复小波滤波所得的复信号 $W T\left(f_{0}, \beta\right)$ 的实部和 虚部互为 Hilbert 变换对, 因此可以直接利用式(7) 的结果获取滤波后信号的包络 $S(t)$

$$
S(t)=\sqrt{[\operatorname{Re}(W T)]^{2}+[\operatorname{Im}(W T)]^{2}}
$$

式中, $\operatorname{Re}$ 和 $\operatorname{Im}$ 分别表示取实部和虚部。复小波滤 波的优势之一就在于滤波和解调可以同步实现。

\section{2 复小波频带优化及改进滤波模型}

式(7)表明复平移 Morlet 小波变换在频域表现 为被分析信号频谱和 Morlet 小波频谱之积, 而从 图 1 中可以看到 Morlet 小波的频谱具有窗口形式, 因此复小波变换的实质就是滤波。要利用该小波变 换进行共振解调, 中心频率 $f_{0}$ 和带宽 $\beta$ 的确定至关
重要。

\section{1 复小波频带优化新指标}

对于以周期性冲击为特征的滚动轴承局部故 障, 其故障特征信号及其包络表现出明显的瞬态冲 击特性。所以共振解调结果的优劣可以通过解调信 号的冲击性强弱进行衡量, 常用的度量指标有峭度

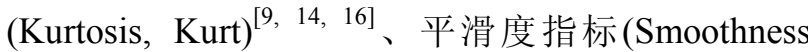
index, SI) ${ }^{[13]}$ 及山农商(Shannon entropy, SE) ${ }^{[17]}$ 等。

设离散时间信号 $\boldsymbol{S}=[s(n), n=1,2, \cdots, n]$ 为复平移 小波滤波所得包络信号, $\boldsymbol{S}$ 的算术均值 $\bar{s}_{\mathrm{A}}$ 、几何均 值 $\bar{s}_{\mathrm{G}}$ 及标准偏差 $\sigma_{V}$ 定义如下

$$
\begin{gathered}
\bar{S}_{\mathrm{A}}=\frac{1}{n} \sum_{n=1}^{N} s(n) \\
\bar{S}_{\mathrm{G}}=\left[\prod_{n=1}^{N} s(n)\right]^{1 / n} \\
\sigma_{V}=\left[\frac{1}{n} \sum_{n=1}^{N} s(n)^{2}-\bar{S}_{\mathrm{A}}\right]^{1 / 2}
\end{gathered}
$$

平滑度指标 SI、峭度 Kurt 和山农熵 SE 定义 如下

$$
\mathrm{SI}=\frac{\overline{s_{\mathrm{G}}}}{\overline{s_{\mathrm{A}}}}
$$

$$
\begin{gathered}
\text { Kurt }=\frac{1}{N} \sum_{n=1}^{N} \frac{\left[s(n)-\bar{s}_{\mathrm{A}}\right]^{4}}{\sigma_{V}{ }^{4}} \\
\mathrm{SE}=-\sum_{n=1}^{N} d_{n} \lg d_{n} \\
d_{n}=\frac{s(n)}{\sum_{n=1}^{N} s(n)}
\end{gathered}
$$

峭度是共振解调技术中使用最为广泛的频带 优化指标, 但是峭度指标只能度量信号的脉冲性(稀 疏性), 而轴承发生故障时的一个典型特征就是这些 冲击是按轴承故障特征频率周期性地发生的，有别 于外界干扰引入的偶然性冲击。下面的例子体现了 引入周期性判断的必要性。

图 2a 是一个轴承内圈故障仿真信号, 采样频率 $20480 \mathrm{~Hz}$, 内圈旋转频率 $15 \mathrm{~Hz}$ ，故障频率 $90 \mathrm{~Hz}$ 。 图 $2 \mathrm{~b}$ 由图 $2 \mathrm{a}$ 部分脉冲构成。图 $2 \mathrm{a}$ 的信号及其包络 的峭度值分别为 34.5 和 22.2 , 而图 $2 \mathrm{~b}$ 的分别为 52.8 和 34.1。后者的两个峭度值分别大于前者, 但显然 前者更符合轴承内圈故障信号特征，因为其周期性 更为明显。所以小波滤波器频带优化时应考虑小波 滤波后所得信号的周期性，以增强特征提取效果。 


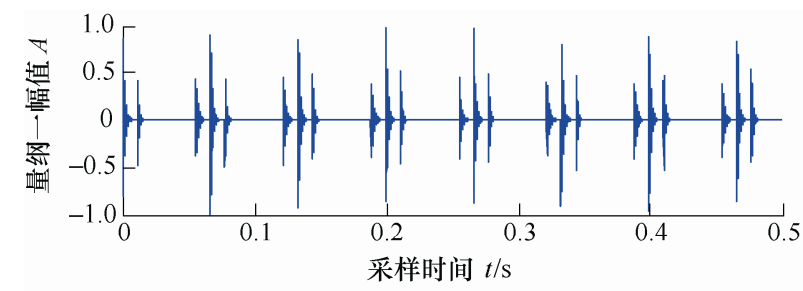

(a) 轴承内圈故障仿真信号

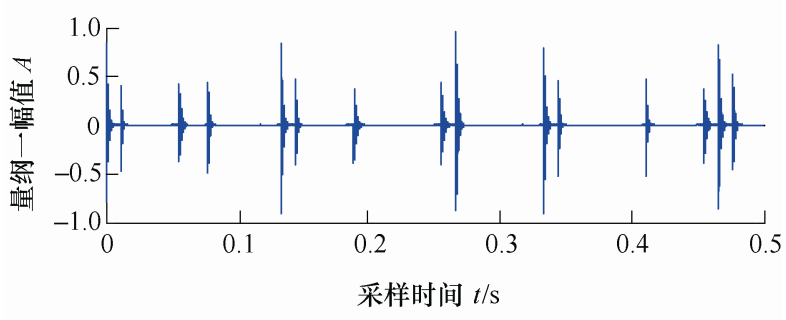

(b) 由图 $\mathrm{a}$ 的部分脉冲构成的仿真信号

图 2 轴承内圈故障仿真信号

为此, QIU 等 ${ }^{[3]}$ 采用奇异值分解 (Singular value decomposition, SVD)来选择周期性最强的小波尺 度。基于 SVD 的周期性判断在构造分解矩阵时易出 现由于采样间隔限制导致的精度问题, 结果往往不 准确 ${ }^{[18]}$ 。本文提出包络谱峰值因子(Crest factor of envelope spectrum, Ec), 该指标考虑了共振频段能量 较大且滤波信号周期性较强的特点

$$
\mathrm{Ec}=\frac{e_{\mathrm{MAX}}}{e_{\mathrm{RMS}}}
$$

式中, $e_{\mathrm{MAX}}$ 和 $e_{\mathrm{RMS}}$ 分别定义为包络谱 $e=\operatorname{FFT}\left(S-\bar{s}_{\mathrm{A}}\right)$ 在 $\left[n \times f_{\mathrm{r}}, f_{\mathrm{s}} / 2\right]$ 范围内的最大值和有 效值, 其中 $f_{\mathrm{r}}$ 和 $f_{\mathrm{s}}$ 分别表示轴承旋转频率及信号采 样频率, 为避免转频对 $\mathrm{Ec}$ 值的影响, $n$ 可取为 2 。 指标 $\mathrm{Ec}$ 越大意味着滤波后信号的信噪比越高且能 量越大。滤波后信号的周期性越好, 该指标越大, 因为在幅值一致的情况下, 周期性越好包络谱的能 量就越大。图 $2 \mathrm{a} 、 2 \mathrm{~b}$ 的信号的 Ec 值分别为 20.3 和 15.9 , 可知所提出的指标能较好地度量信号的周 期性。

\section{2 复小波频带优化方法}

复平移 Morlet 小波滤波器有中心频率 $f_{0}$ 和带宽 $\beta$ 两个参数需要确定, 如果两者分别有 $N_{1}$ 和 $N_{2}$ 个 候选值, 则总共需要评价的参数组合有 $N_{1} \times N_{2}$ 。如 文献[13]将 $f_{0}$ 和 $\beta$ 的取值范围分别离散为 117 和 100 个点, 则需要评价的参数组合有 11700 个, 使得寻 优过程非常缓慢, 不适合仪器执行和现场使用。

滤波器带宽 $\beta$ 如果太小的话, 不能包括足够的 故障特征频率信息。若 $\beta$ 太大, 虽然可以包括更多 故障特征频率的谐波成分, 但也会引入更多噪声干 扰。通常为了确保包络谱分析的可信度, 当存在轴 承故障时, 希望除了找到故障特征频率外, 还能找
到其二阶、三阶甚至更高阶的谐波分量。因此滤波 器带宽 $\beta$ 通常可以设为 3 至 4 倍的最大故障特征频 率 ${ }^{[2,19-21]}$ 。对于滚动轴承而言, 其最大故障特征频 率通常为内圈故障频率(Ball passing frequency of inner race, BPFI), 所以实际上可以固定滤波器带宽 为 $\beta=3 \times \mathrm{BPFI}$ 。但是为了进一步确保解调结果的可 信度, 取 $\beta=[3,3.5,4,4.5,5,5.5,6] \times \mathrm{BPFI}$ 。候选带 宽值只有 7 个, 大大减小了搜索空间。

在确定 $\beta$ 的取值范围之后, 中心频率 $f_{0}$ 要求满 足以下条件 ${ }^{[14,17]}$ 。

（1）根据小波可容许性条件，Morlet 小波频谱 应满足 $\Psi(0)=0$ 。否则，小波将变成一个低通而非 带通滤波器。 $f_{0} / \beta>2.5$ 时, $\Psi(0)<1.7264 \times 10^{-4}$, 近似满足。所以有

$$
f_{0}>2.5 \beta
$$

(2) 为了过滤掉如转子故障等所带来的工频谐 波分量, 小波滤波器的低通截止频率应大于一定倍 数的转子转频 $f_{r}$, 本文取 30 倍

$$
f_{0}-\frac{\beta}{2}>30 \times f_{r}
$$

（3）根据采样定理，小波滤波器的高通截止频 率应小于采样频率 $f_{s}$ 的一半，即

$$
f_{0}+\frac{\beta}{2}<\frac{f_{s}}{2}
$$

(4) 采集轴承故障加速度信号时, 采样频率 $f_{s}$ 通常设置较高以保证充分采样, 而轴承共振频段应 位于传感器线性范围之内, 所以 $f_{0}$ 不宜接近 Nyquist 频率，可取如下

$$
f_{0}<0.8 \times \frac{f_{s}}{2}
$$

综合以上四个条件， $f_{0}$ 的取值范围如下

$$
\begin{gathered}
\max \left(2.5 \beta_{\min }, 30 f_{r}+\frac{\beta_{\min }}{2}\right)<f_{0}< \\
\min \left(\frac{f_{s}}{2}-\frac{\beta_{\min }}{2}, 0.8 \times \frac{f_{s}}{2}\right)
\end{gathered}
$$

通过上述对中心频率和带宽取值范围的分析， 缩小了参数搜索范围，提高了算法效率。以中心频 率为主带宽为辅的频带优化方法对中心频率 $f_{0}$ 的 取值范围进行较为细致的划分，例如 100 点。对于 带宽参数, 通过前面的分析可知, 仅需要粗略划分 即可。 


\section{3 复小波共振解调改进模型}

复小波共振解调改进模型的主要步骤如图 3 所 示。第一步根据轴承的结构参数和转速, 得出轴承 最大的故障特征频率(通常为 $\mathrm{BPFI}$ ), 确定带宽 $\beta$ 的 取值范围。根据最小带宽 $\beta_{\min }$ 与中心频率 $f_{0}$ 之间应 该满足的约束关系式(20), 得出中心频率的取值范 围。将 $f_{0}$ 的取值范围离散化, 利用每组 $\left(f_{0}, \beta\right)$ 对信号 进行包络分析, 计算所得包络信号的包络谱峰值因 子 $\mathrm{Ec}$, 根据 $\mathrm{Ec}$ 最大准则选择最优中心频率和带宽。 最后利用 $\left(f_{0}, \beta\right)$ 的最优参数组合对信号进行共振解 调滤波, 得到滤波信号。进一步可以利用 FFT 作包 络谱分析, 将包络谱上的主要谱峰与轴承故障理论 频率进行对比确定轴承状态及故障类型。

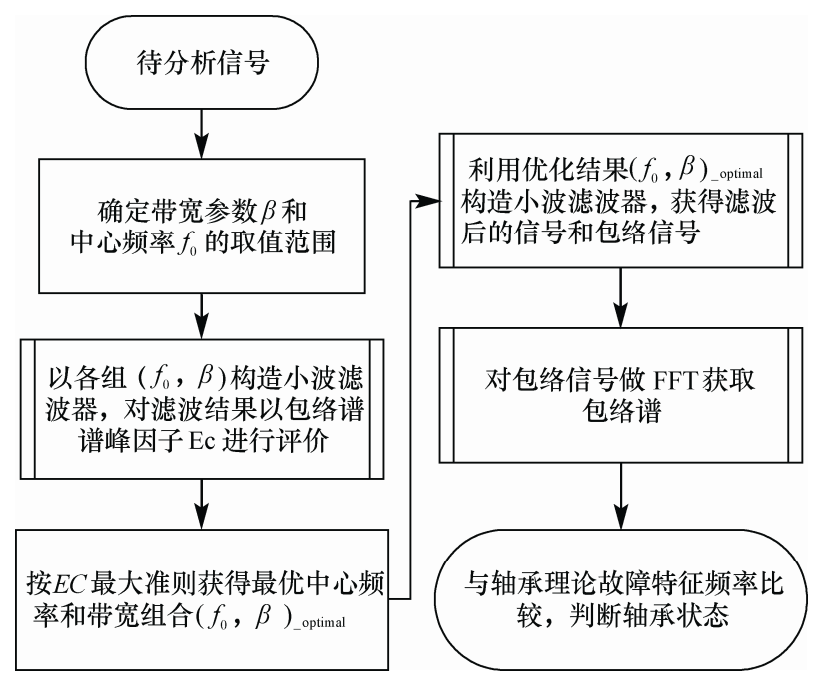

图 3 复小波共振解调改进模型

相比于已有的小波滤波解调模型, 该模型的优 势主要体现在两方面: 一是对两个参数取值范围进 行了限制, 减小参数搜索空间; 二是采用了一个新 的优化指标, 该指标能够同时考虑信号中冲击成分 强弱及其周期性发生的特点。

\section{3 试验台数据分析}

图 $4 \mathrm{a}$ 是滚动轴承疲劳试验台的整体结构示意 图, 图 $4 \mathrm{~b}$ 是试验台局部照片 ${ }^{[3,22-23]}$ 。电动机通过带 传动驱动主轴以 $2000 \mathrm{r} / \mathrm{min}$ 的转速旋转。主轴上安 装有四个型号为 Rexnord ZA-2115 的双列滚柱轴 承, 其中两端的轴承固定在机体上, 中间的两个轴 承通过杜杆机构向主轴施加径向载荷。由转速及轴 承结构参数可知外圈故障特征频率 (Ball passing frequency of outer race, $\mathrm{BPFO})=235.4 \mathrm{~Hz}$, 内圈故 障特征频率 $\mathrm{BPFI}=297.9 \mathrm{~Hz}$ 。疲劳试验于
2004-02-12 的 10:32:39 开始, 2004-02-19 的 06:22:39 试验结束时解体发现轴承 1 发生了严重外圈故障。 试验过程中通过安装在轴承座上的加速度传感器以 $20000 \mathrm{~Hz}$ 的采样频率每隔 $10 \mathrm{~mm}$ 采集振动信号一 次，共采集到 984 个数据文件，每个文件包含 4 列 长为 20480 点的数据, 本文分析的是第二列。

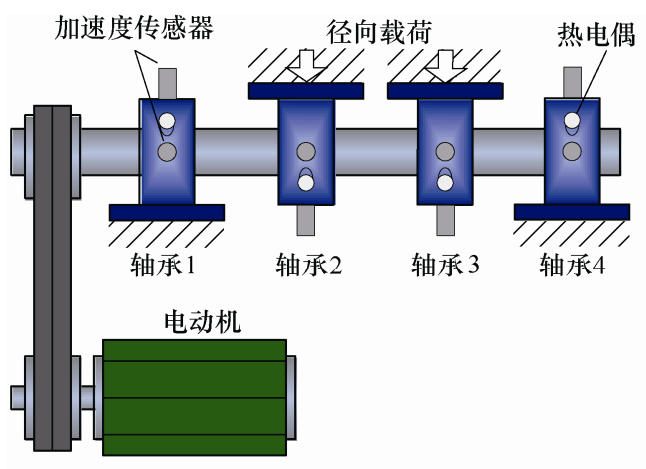

(a) 试验台整体结构示意图

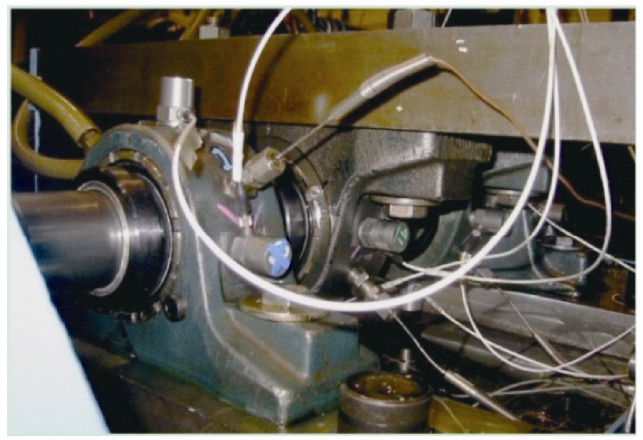

(b) 试验台局部照片

图 4 滚动轴承疲劳试验台

\section{1 晚期故障数据分析}

首先分析 2004-02-19 的 06:02:39 采集的数据文 件的第二列数据, 即 984 个文件中的第 982 个。此 时离最终停机只有 $20 \mathrm{~min}$, 可以认为轴承已经处于 故障晚期。取最小半功率带宽 $\beta_{\min }=3 \times \mathrm{BPFI} \approx 900$ $\mathrm{Hz}$ 。根据式(20)可得中心频率 $f_{0}$ 的取值范围为 [2 250 $\mathrm{Hz}, 8000 \mathrm{~Hz}$, 将该范围离散为 100 个点, 因此总 共有 $100 \times 7=700$ 个 $\left(f_{0}, \beta\right)$ 参数组合。利用不同的优化 指标包括提出的包络谱峰值因子 $\mathrm{Ec}$ 、滤波信号的峭 度 Kurt、滤波后信号包络的平滑度指标 SI 以及滤波 信号的山农熵 SE 对各参数组合进行评价, 各指标 所对应的最优参数组合如表 1 所示。

表 1 不同优化指标获得的最优频带参数(晚期故障)

\begin{tabular}{ccc}
\hline 优化指标 & 最优带宽×BPFI/Hz & 最优中心频率 $/ \mathrm{Hz}$ \\
\hline 包络谱峰值因子 Ec & 3 & 2338.2 \\
信号峭度 Kurt & 6 & 3756.4 \\
包络平滑度 SI & 3 & 2250.0 \\
信号山农熵 SE & 6 & 2907.2 \\
\hline
\end{tabular}


图 5a 给出了原始信号 $0.05 \mathrm{~s}$ 时长的时域波形, 冲击特征不明显。图 $5 b$ 是以 10240 个数据点作 FFT 得到的幅值谱, 可以看到轴承故障冲击引起的共振 调制现象主要存在于三个频段, 即 $500 ~ 1300 \mathrm{~Hz}$, $2000 \sim 3000 \mathrm{~Hz}$ 以及 $4000 \sim 6000 \mathrm{~Hz}$, 边频带间隔为 $230 \mathrm{~Hz}$ 或 $232 \mathrm{~Hz}$, 与 $\mathrm{BPFO}=235.4 \mathrm{~Hz}$ 接近。利用 Ec 最大判据得到的最优滤波器中心频率为 2338.2 $\mathrm{Hz}$ ，与图 $5 \mathrm{~b}$ 频谱中标记的 $2311 \mathrm{~Hz}$ 接近。同时从 图 5b 可以看到 $2000 \sim 3000 \mathrm{~Hz}$ 共振频段的幅值高于 $4000 \sim 6000 \mathrm{~Hz}$ 频段, 正如前面定义 Ec 时所述, Ec 不仅能够提取冲击现象明显的共振频段而且能够提 取冲击能量最大的共振信号, 而冲击能量一方面取 决于单个冲击力度, 另一方面则是冲击发生的密度。 $500 \sim 1300 \mathrm{~Hz}$ 频段也具有较大幅值, 但该频段不在 由式(20)确定的 $f_{0}$ 取值范围内。图 $5 \mathrm{c} 、 5 \mathrm{~d}$ 分别给出 了 Ec 对应的最优滤波器滤波后的时域信号及其包 络谱。可以看到滤波后信号冲击特征得到明显增强 且具有显著的周期性, 而包络谱上的 $230.5 \mathrm{~Hz}$ 频率 成分与 $\mathrm{BPFO}=235.4 \mathrm{~Hz}$ 接近, 并且存在两倍频和三 倍频成分, 其他频率成分及噪声很小, 因而可以判 定轴承已有严重外圈故障。

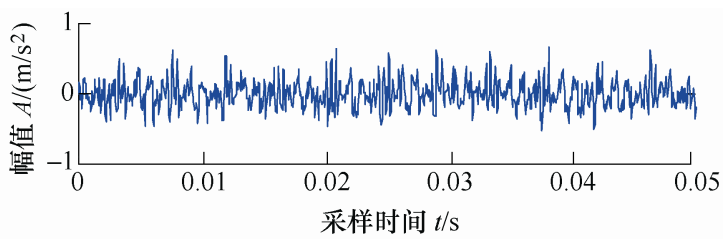

(a) 原始信号

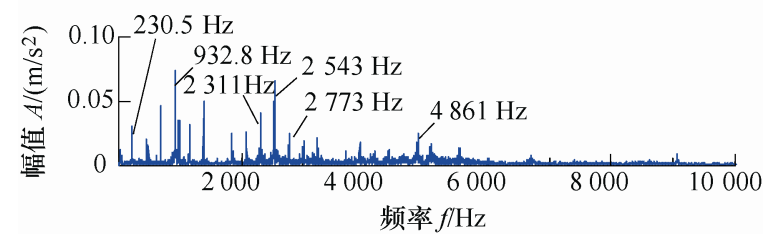

(b) 原始信号频谱

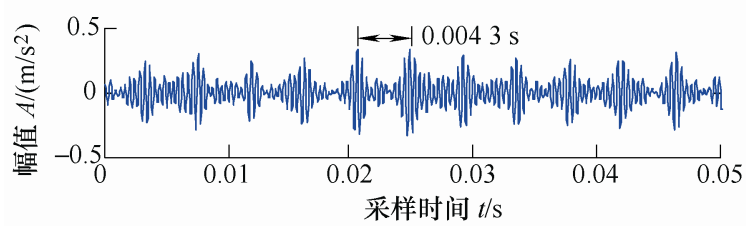

(c) 本文方法获得的滤波信号

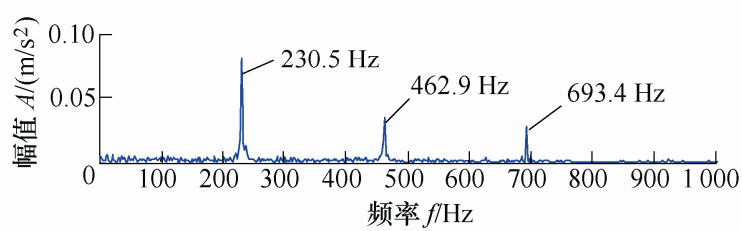

(d) 本文方法获得的包络谱

图 5 原始信号以及本文方法滤波解调结果(晚期故障)

由信号峭度 Kurt、包络平滑度指标 SI 以及信号 山农熵 SE 所对应的最优滤波器得到的滤波信号及 包络谱如图 6 所示。由表 1 可知峭度 Kurt 对应的最
优滤波器中心频率为 $3756.4 \mathrm{~Hz}$, 邻近图 $6 \mathrm{~b}$ 所示的 $4000 \sim 6000 \mathrm{~Hz}$ 共振频段。图 $6 \mathrm{~b}$ 虽然也能得到较清 楚的包络谱，但图 6a 时域周期性冲击特征不如图 $5 \mathrm{c}$ 明显。原因在于峭度只考虑冲击强弱而不考虑冲 击的周期性。平滑度 SI 所得的最优参数与包络谱峰 值因子 $\mathrm{Ec}$ 相近，所以图 6c、6d 的结果与图 5c、5d 类似。由滤波后信号山农熵 SE 最小得到的最优中 心频率 $2907.2 \mathrm{~Hz}$ 位于 $2000 \sim 3000 \mathrm{~Hz}$ 的边沿, 但 $6 \times$ BPFI 的大带宽使滤波器也能覆盖一定阶次的调 制边带, 然而过大的带宽会引入多余的噪声，因此 图 6e 的时域冲击现象不如图 5c 显著。

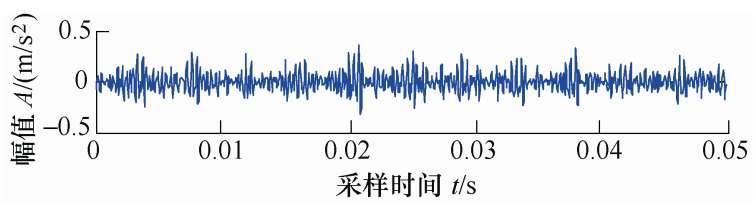

(a) 根据信号峭度 Kurt 最大获得的滤波信号

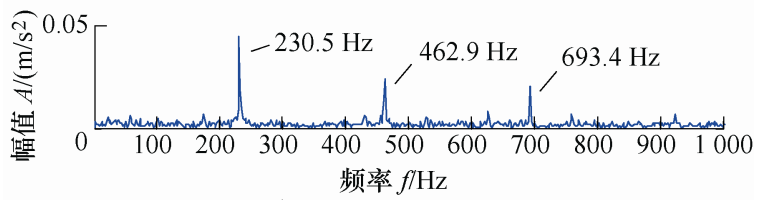

(b) 根据信号峭度 Kurt 最大获得的包络谱

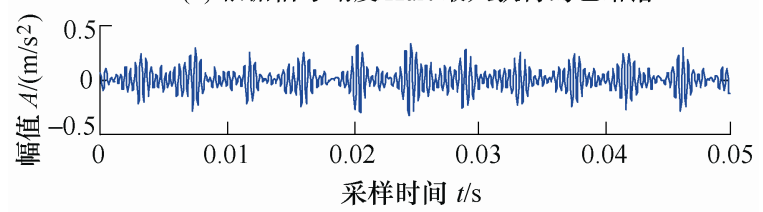

(c) 根据包络平滑度SI最小获得的滤波信号

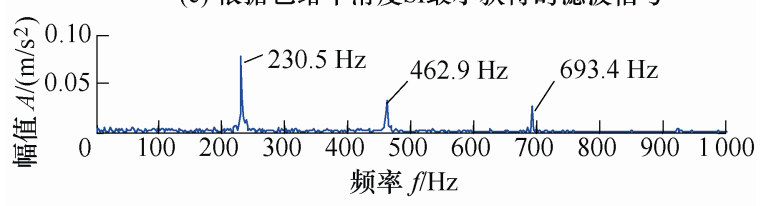

(d) 根据包络平滑度SI最小获得的包络谱

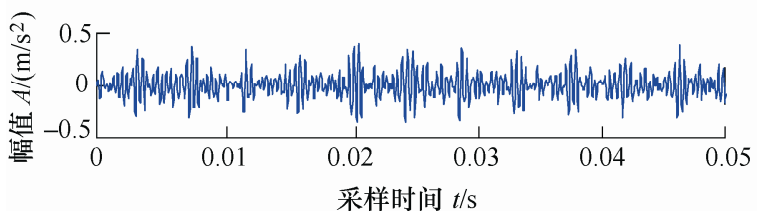

(e) 根据信号山农熵SE最小获得的滤波信号

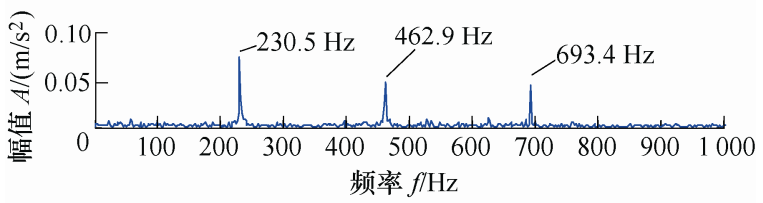

(f) 根据信号山农摘SE最小获得的包络谱

图 6 峭度、平滑度及山农熵滤波解调结果(晚期故障)

\section{2 早期故障数据分析}

早期故障诊断希望在故障萌芽或程度尚轻微 时即能给出正确的诊断结果, 以期为设备维修和生 产计划安排争取足够的缓冲时间和主动性 ${ }^{[24]}$ 。本节 分析的信号采集时间为 2004-02-17 的 07:32:39, 即 984 个数据文件中的第 703 个, 较最后停机时间 
2004-02-19 的 06:22:39 提前近 2 天, 而总的轴承寿 命时间约 7 天，因此可以认为此时处于故障早期阶 段。各优化指标对应的最优频带参数如表 2 所示, 可以看到只有 $\mathrm{Ec}$ 所对应的中心频率 $f_{0}=4552.4 \mathrm{~Hz}$ 位于图 7b 信号频谱所示的 $2000 \sim 6000 \mathrm{~Hz}$ 共振频段。

\section{表 2 不同优化指标获得的最优频带参数(早期故障)}

\begin{tabular}{ccc}
\hline 优化指标 & 最优带宽 $\times \mathrm{BPFI} / \mathrm{Hz}$ & 最优中心频率 $/ \mathrm{Hz}$ \\
\hline 包络谱峰值因子 $\mathrm{Ec}$ & 3 & 4552.4 \\
信号峭度 Kurt & 6 & 7408.0 \\
包络平滑度 SI & 3 & 7503.5 \\
信号山农熵 $\mathrm{SE}$ & 3 & 7503.5 \\
\hline
\end{tabular}

图 7a 给出的是数据文件中第二列数据的时域 波形, 不能看出轴承故障引起的瞬态冲击成分。故 障早期缺陷尺寸较小、冲击力度小, 因此在图 $7 \mathrm{~b}$ 所示的信号频谱及其局部放大图中也没有明显的调 制边带。Ec 对应的最优滤波结果如图 7c、7d 所示, 在时域中已经能够看到冲击成分, 而包络谱中则明 显存在外圈故障特征频率及其谐波成分。因此可以 确定外圈故障已经存在, 但是包络谱中存在较大的 “地毯” 噪声, 故障特征频率成分的幅值也较晚期故 障小很多，因此可以判断轴承处于故障初期阶段。

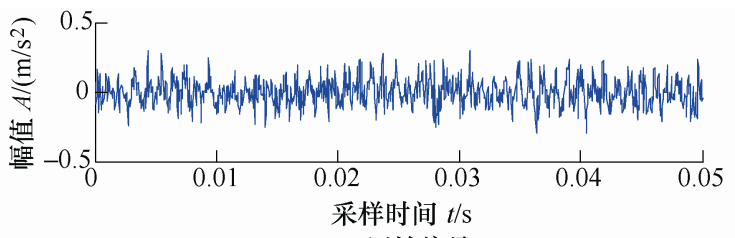

(a) 原始信号

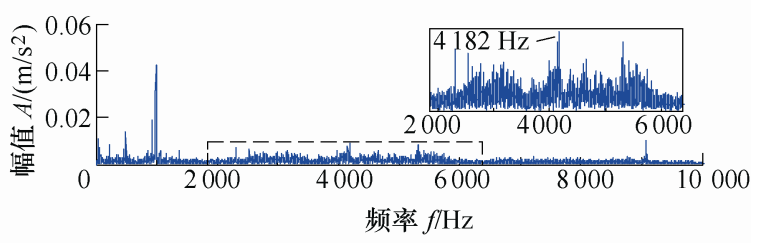

(b) 原始信号频谱

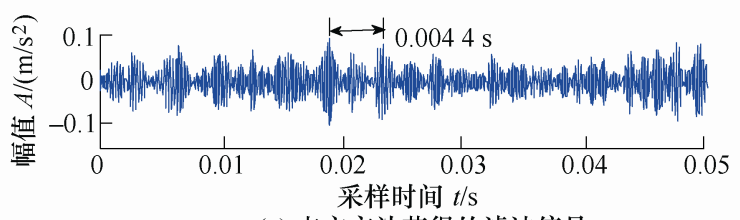

(c) 本文方法获得的滤波信号

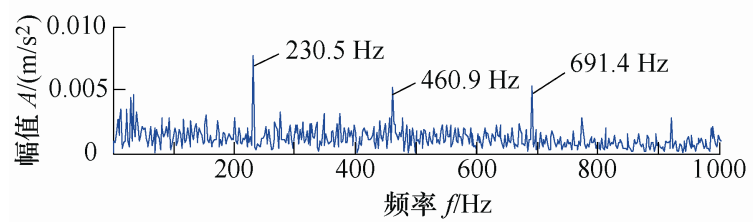

(d) 本文方法获得的包络谱

图 7 原始信号以及本文方法滤波解调结果(早期故障)

其余三个指标结果如图 8 所示, 其中 SI 和 SE 的最优参数组合完全相同, 故未重复画出。可以看 到无论是时域还是频域均不能提取到轴承故障特
征。此例说明了本文所提出的包络谱峰值因子 $\mathrm{Ec}$ 在早期故障诊断方面的优势。

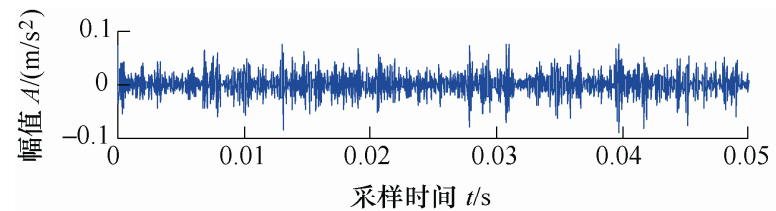

(a) 根据信号峭度 Kurt 最大获得的滤波信号

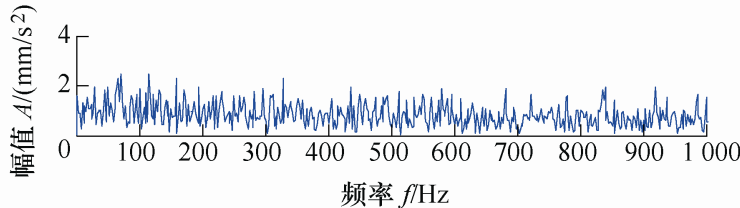

(b) 根据信号峭度 Kurt 最大获得的包络谱

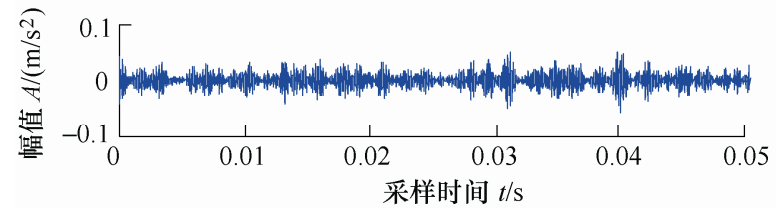

(c) 根据包络平滑度SI或信号山农熵SE最小获得的滤波信号

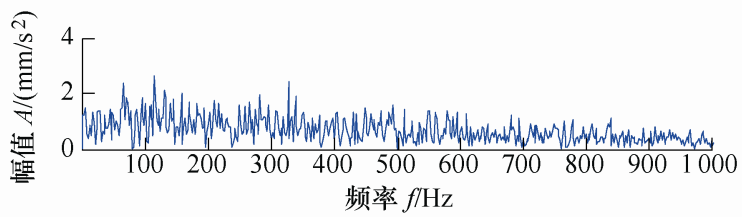

(d) 根据包络平滑度SI或信号山农熵SE最小获得的包络谱

图 8 峭度、平滑度及山农熵滤波解调结果(早期故障)

\section{4 工程应用}

货车滚动轴承早期故障轨边声学诊断系统 (Trackside acoustic detection system, TADS)利用安 装在钢轨两侧的声学传感器采集列车运行状态下的 声音信号，通过对声音信号的分析实现轴承故障检 测 ${ }^{[25]}$ 。图 9 是某 TADS 探测站检测到的内圈故障轴 承的解体结果, 轴承型号为 $197726 \mathrm{TN}$ 。检测时车 速 $38 \mathrm{~km} / \mathrm{h}$ ，信号采样频率 $48000 \mathrm{~Hz}$ 。可知轴承内 圈转速约为 $240 \mathrm{r} / \mathrm{min}$, 内圈故障特征频率约为 44 $\mathrm{Hz}$ 。设置带宽 $\beta$ 取值范围为[3:0.5:6] $\times 44 \mathrm{~Hz}$, 由式 (20) 可知中心频率 $f_{0}$ 的取值范围为 $[1980 \mathrm{~Hz}, 19200$ $\mathrm{Hz}$ ], 将该范围离散为 100 个点。利用复小波共振解 调对该轴承声音信号进行分析，各优化指标得到的 最优中心频率和带宽参数组合如表 3 所示。

表 3 不同优化指标获得的最优频带参数(工程应用)

\begin{tabular}{ccc}
\hline 优化指标 & 最优带宽×BPFI/Hz & 最优中心频率 $/ \mathrm{Hz}$ \\
\hline 包络谱峰值因子 Ec & 3 & 13924.4 \\
信号峭度 Kurt1 & 6 & 18338.7 \\
包络平滑度 SI & 3 & 7667.6 \\
信号山农熵 SE & 3 & 7493.7 \\
\hline
\end{tabular}




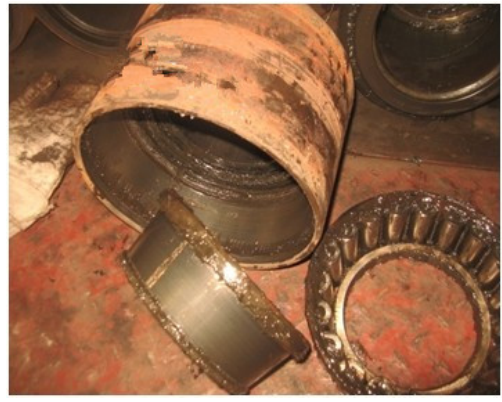

图 9 货车内圈故障轴承

图 10a 给出了原始声音信号的时域波形, 冲击 特征不是非常明显。而图 $10 \mathrm{~b}$ 的频谱中也难于找到 故障特征频率，但频谱中存在一个以 $13115.9 \mathrm{~Hz}$ 为中心的共振频段。表 3 所示的最优滤波参数组合 中只有 Ec 所得的最优中心频率 $13924.4 \mathrm{~Hz}$ 与该共 振频段最为接近, 进一步说明了 $\mathrm{Ec}$ 指标能够提取 到冲击能量最大的共振频段, 而在单次冲击强度一 致的情况下冲击的周期性强弱决定了冲击总能量的 大小。利用 $\mathrm{Ec}$ 对应的最优滤波器进行滤波解调, 结果如图 10c、10d 所示, 可以看到滤波后时域冲击 特征已非常明显, 冲击响应的强度受到转频调制, 包 络谱中也存在内圈故障特征频率及转频调制边带。

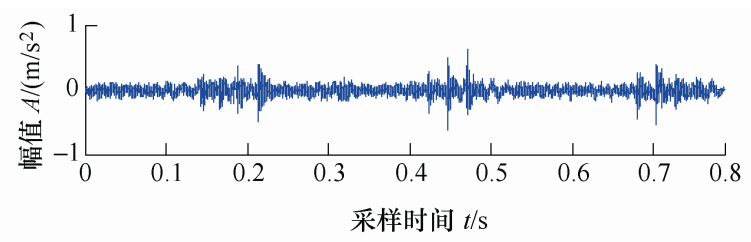

(a) 原始信号

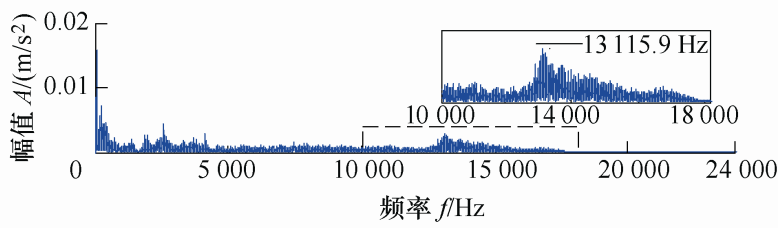

(b) 原始信号频谱

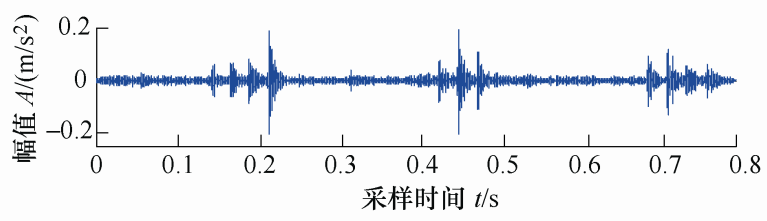

(c) 本文方法获得的滤波信号

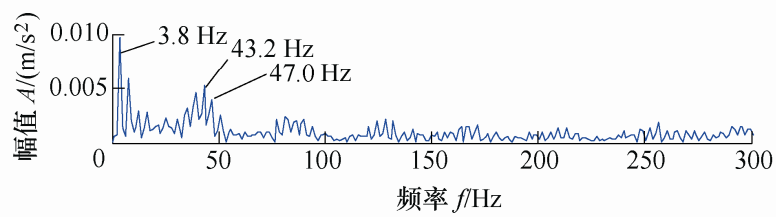

(d) 本文方法获得的包络谱

图 10 原始信号以及本文方法滤波解调结果(工程应用)

由信号峭度 Kurt、包络平滑度指标 SI 以及信号 山农商 SE 所对应的最优滤波器得到的滤波信号如
图 11 所示。与其他指标相比, 由峭度 Kurt 得到的 冲击特征的周期性最弱，反映峭度在提取循环冲击 类故障特征时的缺点，即只考虑单个冲击的强弱而 不能反映冲击发生的周期性。图 11b、11c 所示的 SI 和 SE 的特征提取效果优于 Kurt, 但如图中虚线 标示部分所示，部分冲击特征的提取效果不如图 $10 \mathrm{c}$ 明显。由 Ec、Kurt、SI 及 SE 提取到的脉冲响 应的最大幅值分别为 $0.3984 \mathrm{~m} / \mathrm{s}^{2} 、 0.0458 \mathrm{~m} / \mathrm{s}^{2}$ 、 $0.2372 \mathrm{~m} / \mathrm{s}^{2}$ 和 $0.2330 \mathrm{~m} / \mathrm{s}^{2}$, 其中 $\mathrm{Ec}$ 提取到的冲击 特征强度最大, 从另一方面反映了本文提出的包络 谱峰值因子 $\mathrm{Ec}$ 在提取循环冲击特征中的优势。

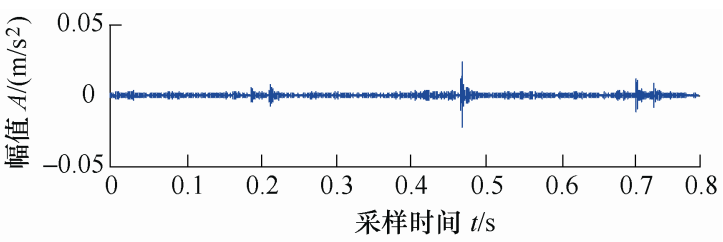

(a)根据信号峭度Kurt最大获得的滤波信号

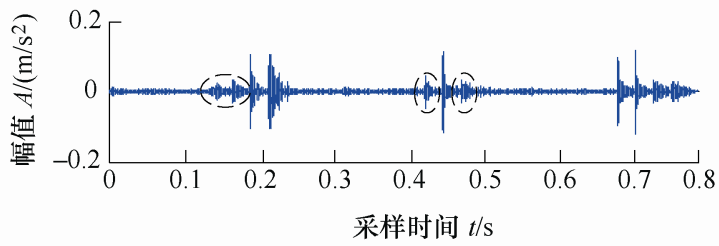

(b)根据平滑度 SI 最小获得的滤波信号

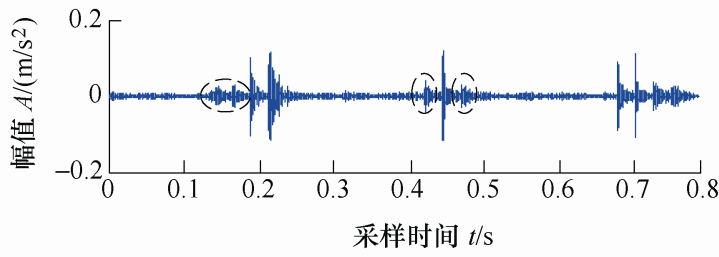

(c)根据信号山农摘 SE 最小获得的滤波信号

图 11 峭度、平滑度及山农熵滤波结果(工程应用)

\section{5 结论}

(1) 针对复平移 Morlet 小波用于循环冲击类故 障特征提取时的频带参数(中心频率和带宽) 优化问 题, 讨论了参数取值对结果的影响并界定了参数的 取值范围。提出以中心频率为主带宽为辅的优化方 案, 即对中心频率取值范围进行较为精细的划分, 而带宽仅取有限的几个值，在保证特征提取效果的 前提下大大减小了参数搜索空间, 提高了效率。

（2）现有的滤波效果评价指标如峭度、平滑度 和山农熵等只能度量信号中冲击成分的强弱, 而不 能反映冲击发生的周期性特点。提出了一个新的频 带优化指标一一包络谱峰值因子, 该指标能够同时 度量冲击及其周期性的强弱。试验台数据及工程应 
用分析表明, 相比于峭度、平滑度及山农熵等指标, 提出的指标能够准确找到故障特征最强的共振 频带。

\section{参 考 文 献}

[1] MCFADDEN P, SMITH D. Model for the vibration produced by a single point defect in a rolling element bearing[J]. Journal of Sound and Vibration, 1984, 98(2): 263-273.

[2] MCFADDEN P, SMITH J. Vibration monitoring of rolling element bearings by the high-frequency resonance technique - a review[J]. Tribology International, 1984, 17(1): 3-10.

[3] QIU Hai, LEE J, LIN Jing, et al. Wavelet filter-based weak signature detection method and its application on rolling element bearing prognostics[J]. Journal of Sound and Vibration, 2006, 289(1): 1066-1090.

[4] RUBINI R, MENEGHETTI U. Application of the envelope and wavelet transform analyses for the diagnosis of incipient faults in ball bearings[J]. Mechanical Systems and Signal Processing, 2001, 15(2): 287-302.

[5] 梁霖, 徐光华. 基于自适应复平移 Morlet 小波的轴承 包络解调分析方法[J]. 机械工程学报, 2006, 42(10): 151-155.

LIANG Lin, XU Guanghua. Optimal envelope demodulation method based on adaptive complex shifted Morlet wavelet in bearing diagnosis[J]. Chinese Journal of Mechanical Engineering, 2006, 42(10): 151-155.

[6] 张中民, 卢文祥, 杨叔子, 等. 基于小波系数包络谱的 滚动轴承故障诊断 [J]. 振动工程学报, 1998, 11(1): 65-69.

ZHANG Zhongmin, LU Wenxiang, YANG Shuzi, et al. Application of the envelop spectrum of wavelet coefficients to fault diagnosis in roller bearing[J]. Journal Vibration Engineering, 1998, 11(1): 65-69.

[7] YIAKOPOULOS C, ANTONIADIS I. Wavelet based demodulation of vibration signals generated by defects in rolling element bearings[J]. Shock and Vibration, 2002, 9(6): 293-306.

[8] 崔玲丽, 康晨晖, 张建宇, 等. 基于时延相关及小波包 系数熵阈值的增强型共振解调方法 [J]. 机械工程学报, 2010, 46(20): 53-57.

CUI Lingli, KANG Chenhui, ZHANG Jianyu, et al. Enhanced resonance demodulation based on delayed correlation and entropy threshold of wavelet packet coefficient[J]. Journal of Mechanical Engineering, 2010,
46(20): $53-57$.

[9] LIN Jing, ZUO Mingjian. Gearbox fault diagnosis using adaptive wavelet filter[J]. Mechanical Systems and Signal Processing, 2003, 20(8): 2022-2045.

[10] YAN Ruqiang, GAO R. Energy-based feature extraction for defect diagnosis in rotary machines[J]. IEEE Transactions on Instrumentation and Measurement, 2009, 58(9): 3130-3139.

[11] 林京, 屈梁生. 基于连续小波变换的信号检测技术与故 障诊断[J]. 机械工程学报, 2000，36(12): 95-100.

LIN Jing, QU Liangsheng. Feature detection and fault diagnosis based on continuous wavelet transform[J]. Chinese Journal of Mechanical Engineering, 2000, 36(12): 95-100.

[12] 何岭松, 李巍华. 用 Morlet 小波进行包络检波分析 [J]. 振动工程学报, 2002, 15(1): 119-122.

HE Lingsong, LI Weihua. Morlet wavelet and its application in enveloping[J]. Journal of Vibration Engineering, 2002, 15(1): 119-122.

[13] BOZCHALOOI I, LIANG M. A smoothness index-guided approach to wavelet parameter selection in signal de-noising and fault detection[J]. Journal of Sound and Vibration, 2007, 308(1-2): 246-267.

[14] HE Wei, JIANG Zhinong, FENG Kun. Bearing fault detection based on optimal wavelet filter and sparse code shrinkage[J]. Measurement, 2009, 89(6): 1066-1090.

[15] NIKOLAOU N, ANTONIADIS I. Demodulation of vibration signals generated by defects in rolling element bearings using complex shifted morlet wavelets[J]. Mechanical Systems and Signal Processing, 2002, 16(4): 677-694.

[16] ANTONI J. The spectral kurtosis: A useful tool for characterising non-stationary signals[J]. Mechanical Systems and Signal Processing, 2006, 20(2): 282-307.

[17] SU Wensheng, WANG Fengtao, ZHU Hong, et al. Rolling element bearing faults diagnosis based on optimal morlet wavelet filter and autocorrelation enhancement[J]. Mechanical Systems and Signal Processing, 2010, 42(7): 1092-1102.

[18] 李建, 刘红星, 屈梁生. 探测信号中周期性冲击分量的 奇异值分解技术 [J]. 振动工程学报, 2002, 15(4): 415-418.

LI Jian, LIU Hongxing, QU Liangsheng. Detection of periodic impulse components in signals using singular value decomposition[J]. Journal of Vibration Engineering, 2002, 15(4): 415-418. 
[19] DJEBALA A, OUELAA N, HAMZAOUI N. Detection of rolling bearing defects using discrete wavelet analysis[J]. Meccanica, 2008, 43(3): 339-348.

[20] NIKOLAOU N, ANTONIADIS I. Rolling element bearing fault diagnosis using wavelet packets[J]. NDT \& E International, 2002，35(3): 197-205.

[21] 梅宏斌, 吴雅, 杨叔子, 等. 用包络分析法诊断滚动轴 承故障[J]. 轴承, 1993(8)：38-40.

MEI Hongbin, WU Ya, YANG Shuzi, et al. Rolling element bearing fault diagnosis using envelope analysis $[\mathrm{J}]$. Bearing, 1993(8): 38-40.

[22] LEE Jay, QIU Hai, YU Gang, et al. Bearing data set from IMS of university of Cincinnati and NASA ames prognostics data repository[EB/OL]. [2010-12-10]. http: //ti.arc.nasa.gov/tech/dash/pcoe/prognostic-data-repository.

[23] QIU Hai, LEE J, LIN Jing, et al. Robust performance degradation assessment methods for enhanced rolling element bearing prognostics $[\mathrm{J}]$. Advanced Engineering Informatics, 2003, 17(1): 127-140.

[24] 胡桥, 何正嘉, 訾艳阳, 等. 基于模糊支持矢量数据描
述的早期故障智能监测诊断[J]. 机械工程学报, 2005, 41(12): $145-150$

HU Qiao, HE Zhengjia, ZI Yanyang, et al. Incipient fault intelligent monitoring and diagnosis based on fuzzy support vector data description[J]. Chinese Journal of Mechanical Engineering, 2005, 41(12): 145-150.

[25] CLINE J, BILODEAU J, SMITH R. Acoustic wayside identification of freight car roller bearing defects[C]// ASME/IEEE, Joint Railroad Conference, April 15-16, 1998, Philadelphia. Piscataway: IEEE, 1998: 79-83.

作者简介: 张龙(通信作者), 男, 1980 年出生, 博士, 讲师。主要研究 方向为故障诊断、工程信号处理及智能算法。

E-mail: longzh@ecjtu.edu.cn

熊国良, 男, 1962 年出生, 博士, 教授。主要研究方向为设备状态检测 与智能诊断。

E-mail: lgxcxx@ecjtu.edu.cn

黄文艺, 男, 1991 年出生。主要研究方向为滚动轴承和齿轮性能退化定 量评估。

E-mail: 15279109865@163.com 